Originally published as:

\title{
Rickerts, V.
}

Identification of fungal pathogens in tissue samples using fluorescence in situ hybridization [Identifizierung von Pilzen in Gewebeschnitten mit Fluoreszenz-in-situ- Hybridisierung] (2013) Pathologe, 34 (6), pp. 528-533.

DOI: $10.1007 / \mathrm{s} 00292-013-1832-8$

This is an author manuscript.

The definitive version is available at: http://link.springer.com/ 
Identifizierung von Pilzen in Gewebeschnitten mit FISH

Identification of fungal pathogens in tissue samples using FISH

Dr. med. Dr. med. habil Volker Rickerts

Erreger von Pilz-, und Parasiteninfektionen und Mykobakteriosen

Robert Koch-Institut

Nordufer 20

13353 Berlin

Tel:+49-30 18754-2862

Fax:+49-30-18754-2614

Emails: rickertsv@rki.de

Deutsche Schlüsselwörter (5): Invasive Pilzinfektion, Aspergillose, Candidose, Histopathologie, FISH, PCR

Englische Schlüsselwörter (5): Invasive fungal infection, Aspergillosis, Candidiasis, Histopathology, mucormycosis, Fluorescent in Situ Hybridization, PCR 
Pilzinfektionen sind trotz Verfügbarkeit neuer Antimykotika mit einer hohen Letalität assoziiert. Aufgrund differenter Empfindlichkeit verschiedener Pilze kommt der Erregeridentifizierung eine entscheidende Bedeutung in der Auswahl effektiver antimykotischer Therapien zu. Neben der Einteilung der Erregermorphologie im Gewebe und der Erregeranzucht werden vermehrt molekularbiologische Verfahren eingesetzt um Mykoserreger aus Organbiopsien zu identifizieren. PCR basierte Verfahren erlauben einen sensitiven Erregernachweis. Allerdings ist mit dem Nachweis fungaler DNA nicht zu unterscheiden ob eine Kolonisation, Kontamination oder invasive Infektion vorliegt. Hybridisierungstechniken werden entwickelt um Pilze durch Hybridisierung synthetischer, mit Fluoreszenzfarbstoffen markierter Oligonukleotide an komplementäre Sequenzen ribosomaler RNA zu identifizieren. Durch Auswahl geeigneter Sonden können Gruppen von Pilzen bis hin zu einzelnen Spezies fluoreszenzmikoskopisch identifiziert und im infektiösen Prozess lokalisiert werden. Sie wurden erfolgreich angewendet um Erreger tiefer Mykosen zu identifizieren.

\section{Englische Zusammenfassung}

Deep fungal infections are associated with significant mortality despite the availability of new antifungal agents. The identification of causative fungi is important to define successful antifungal therapies as agents differ in in vitro susceptibility. Characterization of tissue morphology and cultivation from tissue provide important clues to patient management. Molecular techniques such as PCR-based assays are increasingly being used to identify agents of invasive fungal infections. However, potential contamination limits its use when ubiquitous fungi are targeted. Hybridisation with fluorescently labeled probes targeting the ribosomal RNA of fungi is emerging as an alternative identification strategy. Using conserved or variable regions of the rRNA as targets, group- or species specific probes can be synthesized to identify fungal pathogens and localize them in the infectious process. They have been successfully applied to deep fungal infections due to different agents in various organ samples.

\section{Hinführung zum Thema}

Pilzinfektionen sind trotz Verfügbarkeit neuer Antimykotika mit einer hohen Letalität assoziiert. Aufgrund differenter Antimykotikaempfindlichkeit kommt der Erregeridentifizierung eine entscheidende Bedeutung in der Auswahl effektiver Antimykotika zu. Hierfür werden vermehrt molekularbiologische Verfahren an Gewebeproben angewendet. Hybridisierungstechniken wie die Fluoreszenz in situ Hybridisierung (FISH) werden entwickelt um Pilze nach Hybridisierung spezifischer Sonden im infizierten Gewebe zu identifizieren und zu lokalisieren um die kausale Bedeutung nachgewiesener Pilze zu sichern. 
Invasive Pilzinfektionen durch opportunistische Pilze sind typische Komplikationen bei der wachsenden Zahl Immunsupprimierter wie Krebspatienten, Transplantatempfänger oder kritisch Kranke. Die Diagnose invasiver Mykosen ist in Frühstadien aufgrund unspezifischer klinischer Zeichen meist schwierig. Ein kultureller Erregernachweis ist oft nicht möglich. Gelingt die Anzucht von Pilzen aus primär nicht sterilen Kompartimenten, so ist stets sorgfältig abzuwägen ob es sich um den verursachenden Erreger handelt, da Pilze in der Umwelt weit verbreitet sind und Haut und Schleimhäute mit Pilzen kolonisiert sein können. Daher kommt dem Nachweis von Pilzelementen im Gewebe die entscheidende Bedeutung bei der Sicherung der Diagnose einer invasiver Mykosen zu [3].

Pathologen werden häufig um eine Klassifizierung von Pilzelementen die im Gewebe nachweisbar sind gebeten um Rückschlüsse auf den Erreger einer Mykose zu ziehen. Dies ist insbesondere dann unerlässlich, wenn die kulturelle Anzucht nicht versucht wurde bzw. nicht gelingt oder Pilze mit unklarem pathogenen Potential angezüchtet werden. Die Identifizierung von Pilzelementen im Gewebe ist die entscheidende Basis für eine rationale Auswahl von Antimykotika. Sie kann weiterhin helfen weiterführende Diagnostik, z.B. serologische oder molekularbiologische Tests gezielt einzusetzen [8].

Identifizierung von Pilzelementen in Gewebeschnitten

In Gewebeschnitten können Pilzelemente als Hefen oder Hyphen klassifiziert werden. Schimmelpilze können weiter differenziert werden in Melanin produzierende Schwärzepilze, die selten septierten Hyphen der Erreger von Mucormykosen sowie septierte Hyphen wie sie z.B. von Aspergillus, Scedosporium, Fusarium und vielen anderen Schimmelpilzen gebildet werden [13]. Schwierigkeiten kann diese Klassifizierung vor allem bei nur vereinzelt nachweisbaren Pilzelementen im Gewebe machen. Dann ist auch eine Unterscheidung zwischen Hyphen oder Pseudohyphen von Candida und septierten Schimmelpilzhyphen bzw. deren Abgrenzung von den selten septierten Hyphen bei Mucormykose unter Umständen nicht sicher möglich. Auch die Melaninisierung von Hyphen kann schwach ausgeprägt sein, womit Erreger von Schwärzepilzinfektionen nicht sicher erkannt werden können.

Da Pilzelemente von Mykoseerregern im Gewebe pleomorph erscheinen können ist auch die histopathologische Verdachtsdiagnose von Mischinfektionen schwierig. In einzelnen Serien wurde bei 35\% histologisch dokumentierter Mykosen eine Mischinfektion vermutet [12]. Aufgrund der wenigen sicher unterscheidbaren morphologischen Charakteristika von Pilzen im Gewebe könnte es sich hier um eine vorsichtige Schätzung der tatsächlichen Inzidenz von Mischinfektionen handeln.

Zunehmende klinische Bedeutung von „exotischen Pilzen“

Etwa 100 der 1.5 Millionen postulierten Pilzarten wurden als Erreger invasiver Mykosen beschrieben. Kulturelle Erregernachweise dokumentieren neben den drei häufigen opportunistischen Mykoseerregern Aspergillus, Candida und Cryptococcus die Bedeutung von vormals als Exoten eingestuften Pilzen als Erreger invasiver Infektionen. In einer prospektiven multizentrischen Kohortenstudie von Patienten mit hohem Risiko für Pilzinfektionen nach Knochenmarktransplantation kommt mittlerweile eine Infektion durch diese „exotischen“ Schimmelpilze auf drei Patienten mit invasiver 
Aspergillose [10]. Prominentestes Beispiel unter diesen Mykosen ist die Mucormykose. Klinisch sind diese Infektionen nicht sicher von anderen Schimmelpilzinfektionen zu unterscheiden. Die verursachenden Pilze sind durch eine primäre Resistenz gegen verschiedene Antimykotika charakterisiert. Infektionen verlaufen unbehandelt tödlich [14]. Die Mucormykose ist zu einem Paradigma für die Wichtigkeit des schnellen Erregernachweises und gezielter antimykotischer Therapien bei Patienten mit Schimmelpilzinfektionen geworden (siehe Kapitel „PCR-Diagnostik von Mucormykosen" in diesem Heft). Bei Patienten mit Mucormykose, bei denen innerhalb von 6 Tagen nach Diagnose eine wirksame Therapie begonnen wurde, konnte in einer retrospektiven Kohortenstudie eine Reduktion der Letalität von 83 auf 49\% dokumentiert werden [2]. Für weitere Infektionen durch Schimmelpilze mit erworbener oder intrinsischer Resistenz gegen Antimykotika wie Fusarium oder Scedosporium gilt vermutlich entsprechendes. Die Epidemiologie von Infektionen durch diese Pilze ist nicht gut verstanden, da Hyphen dieser Erreger im Gewebe nicht sicher von Aspergillus unterscheidbar sind. Die Bedeutung von Infektionen durch in vitro resistente Pilze kann sich durch den zunehmenden Einsatz prophylaktischer Azolantimykotika mit Wirksamkeit gegen Aspergillus fumigatus, wie sie bei Hochrisikopatienten häufig durchgeführt wird, weiter verstärken. Daher ist insbesondere bei Versagen antimykotischer Prophylaxen, bei Durchbruchinfektionen unter antimykotischer Therapie und ausbleibender Besserung auf empirische Antimykotikatherapien die Identifizierung verursachender Pilze auf Genus-, oder besser auf Speziesebene ein entscheidender Baustein für den optimalen Einsatz antimykotischer Therapiestrategien [15].

\section{Molekulare Techniken zur Identifizierung von Pilzen}

Der Nachweis ribosomaler RNA-Gene von Mikroorganismen hat die Bedeutung der Diversität von Mikroorganismen zunächst in der Umweltmikrobiologie entscheidend geprägt. Die Mehrzahl der mit diesen Techniken nachweisbaren Erreger lassen sich mit Standardtechniken nicht kultivieren[1]. Der Nachweis von ribosomalen RNA (rRNA)-Genen nach Amplifikation mittels PCR wurde auch erfolgreich zur Identifizierung von Pilzen in Gewebeproben von Patienten mit invasiven Mykosen eingesetzt. Konservierte Areale können mit sog. "broadrange-assays" zum Nachweis einer großen Zahl von Pilzen benutzt werden. Bei Amplifikation variabler Regionen ist eine selektive Amplifikation der rRNA-Gene bestimmter Pathogene möglich. Verschiedene derartiger nicht broadrange Tests müssen allerdings kombiniert werden um die häufigsten Erreger invasiver Mykosen zu erfassen. Aus frischem Biopsiematerial von Patienten mit vermuteter Mykose kann mit diesen Amplifikationstechniken ein sensitiverer Erregernachweis möglich sein als mittels Erregeranzucht [19]. Auch aus Formalinfixierten, Paraffin-eingebetteten Gewebeschnitten kann ein Nachweis von Pilz-DNA noch in bis zu 80\% der Proben von Patienten mit invasiver Mykose möglich sein [16]. Hauptlimitationen PCR basierter Tests, insbesondere von broadrange assays, kann die Anfälligkeit für Kontaminationen durch ubiquitär vorhandene Pilzsporen sein [9]. Auch ist eine Unterscheidung zwischen Kolonisation und Infektion nicht möglich wenn mit Pilzen kolonisierte Schleimhaut in der Probe vorhanden ist. Eine potentielle Limitation amplifikationsbasierter Verfahren ist die präferentielle Amplifikation einzelner Sequenzen, wenn mehrere Zielsequenzen in einer Probe vorkommen. Dies kann zu einer Unterschätzung von Mischinfektionen führen und damit die Auswahl erfolgversprechender Therapien erschweren [16]. 
Identifizierung von Pilzen durch in situ Hybridisierung

Der Erregernachweis mittels in situ Hybridisierung basiert auf der spezifischen Bindung kurzer synthetischer DNA-Sonden an komplementäre Areale der rRNA in Ribosomen von Mikroorganismen (Abb.1). Aufgrund der großen Anzahl der rRNA-Zielmoleküle in lebenden Organismen ist im Gegensatz zu PCR basierten Verfahren zum Nachweis von DNA keine vorherige Amplifikation der Zielstrukturen notwendig wodurch Kontaminationen ohne Bedeutung sind.

Durch Markierung der Sonden mit Fluoreszenzfabstoffen (Fluoreszenz in situ Hybridisierung: FISH) oder anderen Detektionssystemen ist nach Entfernung ungebundener Sonden die Hybridisierung mikroskopisch direkt in Mikroorganismen darstellbar. Entscheidend für das resultierende Fluoreszenzsignal sind vor allem die Penetration der Sonden in mikrobielle Zellen, die Anzahl der Ribosomen pro Zelle, die gewählten Hybridisierungsbedingungen sowie der gewählte Zielabschnitt der rRNA. Nur bei etwa 30\% der rRNA-Abschnitte, meist in phylogenetisch konservierten Bereichen, ist mit einem guten Fluoreszenzsignal zu rechnen. Dies ist insbesondere daher wichtig um das Signal von Autofluoreszenz zu unterscheiden über die bei Pilzen in Gewebeschnitten berichtet wurde $[5,7]$.

Seit seiner Einführung in die Mikrobiologie in den späten 1980'er Jahren wurde Fluoreszenz in situ Hybridisierung benutzt, um phylogenetisch definierte Organismen in Populationen zu lokalisieren, zu identifizieren und zu quantifizieren [4]. Diese spezifischen Fähigkeiten der Methode lassen einen Einsatz an der Schnittstelle zwischen diagnostischer Mikrobiologie und Histologie sinnvoll erscheinen.

Zur Erregerdiagnostik von Mykosen aus Gewebeschnitten wurden zunächst DNA-Sonden benutzt um rRNA von Pilzen mittels in situ Hybridisierung identifizieren. Es wurden Organbiopsien von Patienten mit kulturell gesicherten Mykosen untersucht. Hiermit war eine Hybridisierung in $60-80 \%$ tiefer Mykosen verschiedener Organe möglich. Mittels spezifischer Sonden war eine Zuordnung zu Gruppen von Pilzen wie Mucorales oder Schwärzepilzen oder die Identifizierung einzelner pathogener Arten wie z.B. Histoplasma capsulatum in Übereinstimmung mit dem kulturellen Erregernachweis möglich [16]. Heute werden verschiedene Ansätze benutzt um Hybridisierungsverfahren zur Identifizierung von Pilzen in Gewebeschnitten von Patienten mit gesicherter Mykose weiter zu entwickeln und Determinanten für eine positive Hybridisierung zu evaluieren. Erstens, durch die zunehmende Verfügbarkeit validierter rRNA-Gen Sequenzen ist heute eine bessere Vorhersage möglich welche Erreger mit einer Sonde erfasst werden könnten [1]. Zweitens, die experimentelle Validierung von Sonden mit angezüchteten Pilzen ermöglicht die Dokumentation der Hybridisierung bei verschiedenen Pilzen und erlaubt einen einfachen Vergleich der Signalintensität verschiedener Sonden [17]. Drittens, chemisch modifizierte Sonden (PNA: peptide nucleic acids, LNA: locked nucleic acids) werden anstatt klassischer DNA-Sonden evaluiert um ggf. die Intensität des Hybridisierungssignals zu steigern [13]. Viertens, die Markierung von unterschiedlichen Sonden mit differenten Fluoreszenzfarbstoffen ermöglicht die zeitgleiche Anwendung verschiedener Sonden (Abb.2). Mit einer Kombination von zwei spezifischen, differentiell markierten Sonden konnte eine Mischinfektion durch verschiedene Pilze dokumentiert werden (Abb.3). Durch Kombination einer spezifischen Sonde z.B. für Erreger von Aspergillosen und einer differentiell markierten unspezifischen Sonde wäre die Inzidenz von Mischinfektionen bei Aspergillose systematisch zu untersuchen. Fünftens, neben der mittels FISH darstellbaren rRNA können weitere zelluläre Bestandteile von Pilzen fluoreszenzmikroskopisch dargestellt werden, z.B. doppelsträngige DNA im Zellkern. Hiermit könnten Rückschlüsse auf die Vitalität von Pilzen im infektiösen Geschehen möglich werden. Ribosomale RNA-Zielsequenzen (Ribosomen) und damit das 
mit FISH nachweisbare Fluoreszenzsignal ist von der Stoffwechselaktivität einer Zelle abhängig. Während bei Verfügbarkeit von Nahrungsstoffen etwa $80 \%$ der RNA-Synthese pro-, und eukaryontischer Zellen auf ribosomale RNA entfallen, wird dies bei Mangelbedingungen deutlich weniger [21]. In Stresssituationen können Pilze Zellorganellen wie Ribosomen und bei mehrkernigen Schimmelpilzen auch Zellkerne mittels Makroautophagie in Vakuolen überführen, dort abbauen und dadurch Nährstoffe gewinnen[11, 20]. Derartige mittels Fluoreszenzmikroskopie fassbare strukturelle Veränderungen in Pilzelementen im Gewebe könnten damit genutzt werden um über die Vitalität von Pilzen im Gewebe Aussagen zu treffen. In Übereinstimmung damit konnte im oft nekrotischen Zentrum von Aspergillomen meist keine ribosomale RNA mit Hybridisierungsmethoden nachgewiesen werden, während diese in Arealen am Übergang zu vitalem Gewebe oft möglich ist [13]. Aussagen zur Vitalität von Pilzen in Gewebeproben könnten helfen Therapieentscheidungen bei antimykotisch vorbehandelten Patienten zu rationalisieren. Nicht selten sind Pilzinfektionen, insbesondere die Mucormykosen nach Beginn antimykotischer Therapien progredient trotz wirksamer Therapie. Eine Einschätzung der Vitalität von Pilzen könnte helfen Rückschlüsse auf die Wirksamkeit einer Therapie zu ziehen [14].

Synergistische Informationen durch Kombination molekularer Detektionsstrategien

Die Kombination molekularer Amplifikations- und Hybridisierungsmethoden ermöglicht es die Ätiologie invasiver Mykosen anhand von Formalin-fixierten, Paraffin-eingebetteten Schnitten von Patienten mit gesicherten Pilzinfektionen detailliert zu untersuchen. PCR basierte Methoden können Pilz-DNA sensitiv nachweisen und Pilze bis auf Genus oder sogar Speziesebene identifizieren. Mittels FISH lassen sich bisher nur wenige Gruppen pathogener Pilze unterscheiden aber mit Entwicklung weiterer Sonden ist zu rechnen. Bislang schwer zu untersuchende Entitäten wie Mischinfektionen können dokumentiert werden [18]. Die Möglichkeit zur Lokalisation im Infektionsprozeß könnte helfen, die Ätiologie von Infektionen nachzuweisen die aus einer Schleimhautkolonisation mit verschiedenen Erregern entstehen wie z.B. bei Schimmelpilzinfektionen von Patienten mit zystischer Fibrose oder bei invasiver Candidose mit Erregereintritt im Gastrointestinaltrakt. Die Kombination dieser Methoden wurde auch benutzt um Infektionen durch nicht anzüchtbare Erreger zweifelsfrei zu dokumentieren und die Erreger phylogenetisch zu charakterisieren [6].

Das Ziel der kombinierten Anwendung ist es über ein Verständnis der Ätiologie erstens, Behandlungsstrategien wie empirische antimykotische Therapien zu optimieren, zweitens die Therapie von Patienten mit Durchbruchinfektionen zu optimieren und drittens verlässliche Aussagen zur Ätiologie zu erlangen um Befunde diagnostischer Tests aus einfach zu gewinnenden Proben wie Blut oder BAL zu verstehen. 
Fazit für die Praxis

Molekulare Verfahren werden additiv zu kulturellen und histopathologischen Untersuchungen angewendet um Erreger von Systemmykosen zu identifizieren als Grundlage erfolgreicher antimykotischer Therapien. Fluoreszenz in situ Hybridisierung wird entwickelt um eine Erregeridentifizierung über die Morphologie der Pilzelemente hinaus durch Hybridisierung spezifischer Sonden an Abschnitte der ribosomalen RNA in Pilzen zu ermöglichen. Im Gegensatz zu PCR-basierten Verfahren besteht keine Kontaminationsgefahr. Eine Erregerlokalisation im Infektionsgeschehen ist möglich. Sinnvolle Anwendungen der Technik bestehen bei Verdacht auf Mischinfektionen, bei differierenden Befunden zwischen Histologie und Kultur sowie bei Anzucht ungewöhnlicher Pilze mit undefiniertem pathogenem Potential. 


\begin{tabular}{|c|c|}
\hline Candida 317 & $\begin{array}{l}\text { СACG TACT T T T TCAC TC TC } \\
\text { Candida } 317\end{array}$ \\
\hline $\begin{array}{ll}\text { C.albicans } & \text { A } \\
\text { C.glabrata } & \text { A } \\
\text { A.fumigatus } & \text { A } \\
\text { F.oxysporum } & \text { A } \\
\text { C.neoformans } & \text { A } \\
\text { R.oryzae } & \text { A } \\
\text { S.apiospermum } & \text { A } \\
\text { A.corymbifera } & \text { G } \\
\text { S.prolificans } & \text { G }\end{array}$ & 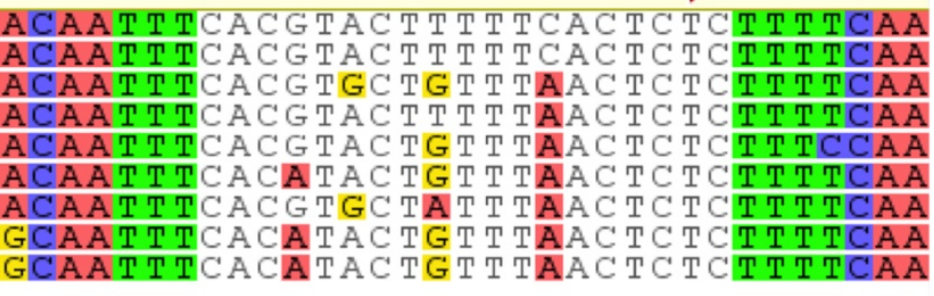 \\
\hline A & AspF \\
\hline $\begin{array}{l}\text { A.fumigatus } \\
\text { F.oxysporum } \\
\text { C.neoformans } \\
\text { R.oryzae } \\
\text { C.albicans } \\
\text { C.glabrata } \\
\text { S.apiospermum } \\
\text { A.corymbifera } \\
\text { S.prolificans }\end{array}$ & 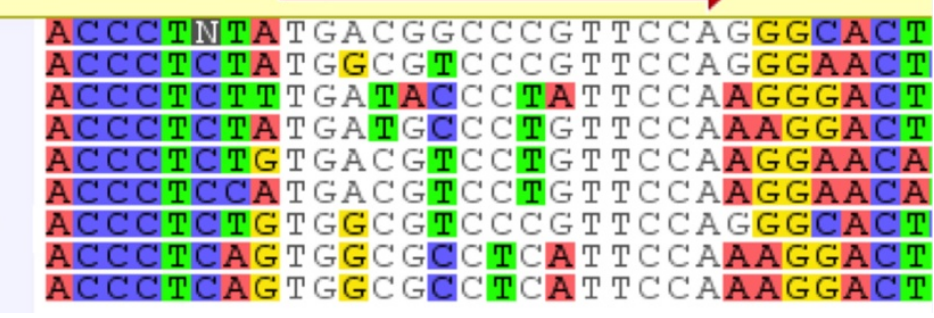 \\
\hline
\end{tabular}

Abbildung 1: Sequenzen von Sonden zur Hybridisierung mit ribosomaler RNA von Pilzen. Alignment von Sequenzen der Sonden Candida 317 (oben), und Aspergillus F (unten) mit Sequenzen der 28S ribosomalen RNA einiger fakultativ pathogener Pilze. Solche kurzen DNA-Sonden können spezifisch mit der rRNA von Pilzen hybridisieren. Fehlpaarungen im Bereich der Sondenbindungsstellen machen die Hybridisierung mit unwahrscheinlich bei stringenten Hybridisierungsbedingungen. Nach [17]. 

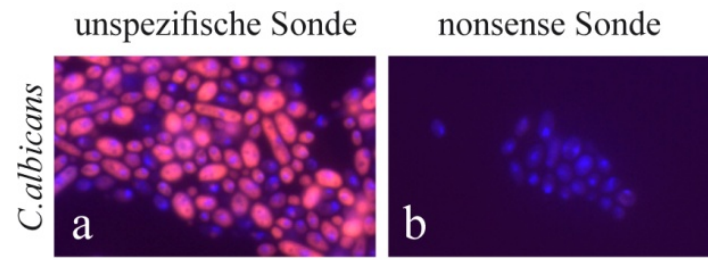

spezifische Sonden
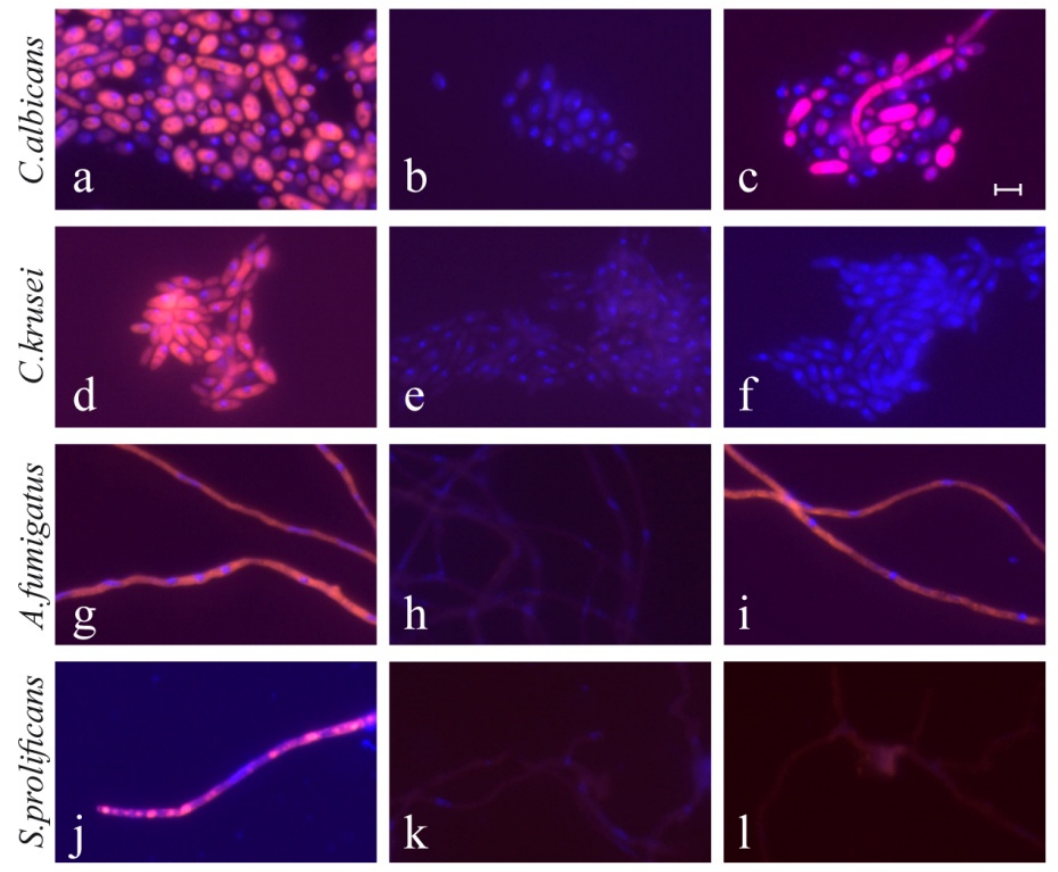

Abbildung 2: Experimentelle Evaluation der Hybridisierung von differentiell markierten FISH-Sonden mit Keimlingen kultivierter Pilze. Hybridisierung mit der unspezifischen eukaryontischen Sonde (EUK 516) markiert mit Cy3 (orange kodiert) und Cy5 (rot kodiert) dokumentiert die erfolgreiche Hybridisierung der verwendeten Pilze $(a, d, g, j)$. Gegenfärbung doppelsträngiger DNA mit DAPI. Die Hybridisierung mit der nonsense-Sonde (nonEUB) markiert mit Cy3 (orange kodiert) und Cy 5 (rot kodiert) schließt Autofluoreszenz der Pilze aus (b,e,h,k). Hybridisierung mit den Sonden Cand 317 markiert mit Cy5 (rot kodiert) und AspF markiert mit Cy3 (orange kodiert) ermöglicht die Unterscheidung verschiedener Pilze wie Candida albicans (c) und Aspergillus fumigatus (i) von nicht erfassten Pilzen wie Candida krusei (f) und Scedosporium prolificans (I). Balken entspricht $5 \mu \mathrm{m}$. Aus [18]. 


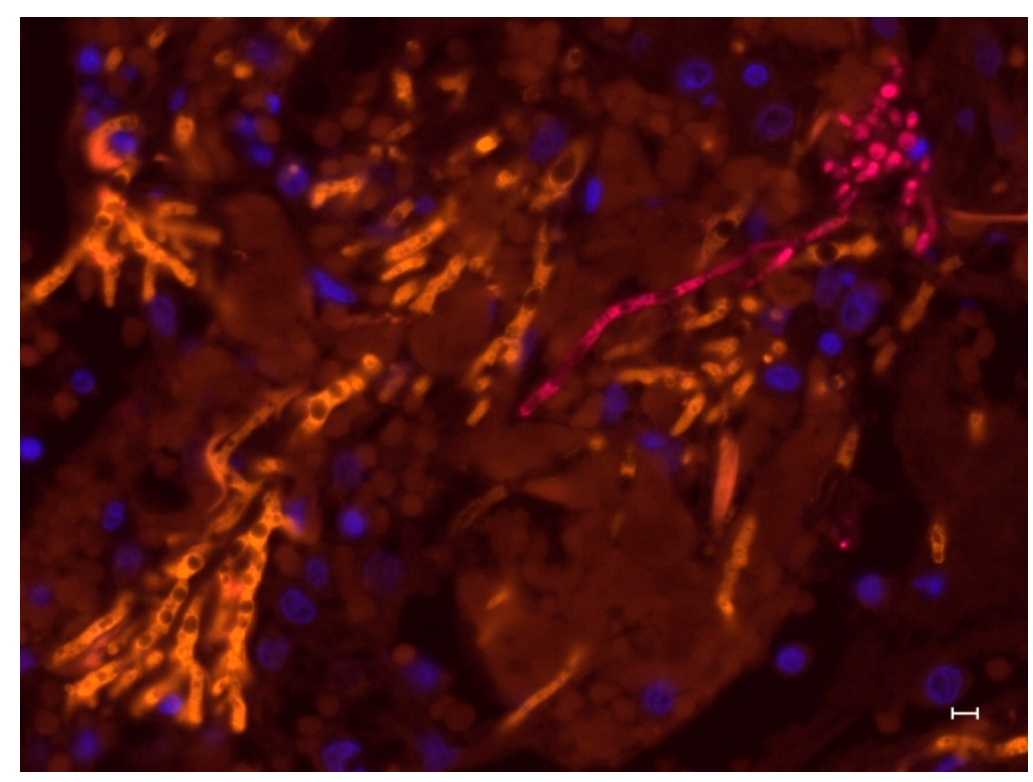

Abbildung 3: Dokumentation einer Mischinfektion durch A.fumigatus und C.albicans. Hybridisierung einer Lungenbiopsie mit den Sonden Asp F markiert mit Cy3 (orange kodiert) zum Nachweis von Erregern der Aspergillose und Cand 317 markiert mit Cy5 (rot kodiert) zum Nachweis häufiger Erreger einer Candidiasis. Gegenfärbung doppelsträngiger DNA mit DAPI. Balken entspricht $5 \mu \mathrm{m}$. Die Erregeridentifizierung wurde mittels broadrange PCR und Sequenzierung bestätigt. Aus [18]. 
Literatur

1. Amann R, Fuchs BM (2008) Single-cell identification in microbial communities by improved fluorescence in situ hybridization techniques. Nature reviews Microbiology 6:339-348

2. Chamilos G, Lewis RE, Kontoyiannis DP (2008) Delaying amphotericin B-based frontline therapy significantly increases mortality among patients with hematologic malignancy who have zygomycosis. Clinical infectious diseases 47:503-509

3. De Pauw B, Walsh TJ, Donnelly JP, Stevens DA, Edwards JE, Calandra T, Pappas PG, Maertens J, Lortholary O, Kauffman CA, Denning DW, Patterson TF, Maschmeyer G, Bille J, Dismukes WE, Herbrecht R, Hope WW, Kibbler CC, Kullberg BJ, Marr KA, Munoz P, Odds FC, Perfect JR, Restrepo A, Ruhnke $M$, Segal BH, Sobel JD, Sorrell TC, Viscoli C, Wingard JR, Zaoutis T, Bennett JE, European Organization for $R$, Treatment of Cancer/Invasive Fungal Infections Cooperative $G$, National Institute of A, Infectious Diseases Mycoses Study Group Consensus G (2008) Revised definitions of invasive fungal disease from the European Organization for Research and Treatment of Cancer/Invasive Fungal Infections Cooperative Group and the National Institute of Allergy and Infectious Diseases Mycoses Study Group (EORTC/MSG) Consensus Group. Clinical infectious diseases 46:1813-1821

4. DeLong EF, Wickham GS, Pace NR (1989) Phylogenetic stains: ribosomal RNA-based probes for the identification of single cells. Science 243:1360-1363

5. Elston DM (2001) Fluorescence of fungi in superficial and deep fungal infections. BMC microbiology 1:21

6. Fredricks DN, Jolley JA, Lepp PW, Kosek JC, Relman DA (2000) Rhinosporidium seeberi: a human pathogen from a novel group of aquatic protistan parasites. Emerging infectious diseases 6:273-282

7. Graf B, Gobel UB, Adam T (1998) Qualitative and quantitative studies of autofluorescence in fungi. Mycoses 41 Suppl 1:39-46

8. Guarner J, Brandt ME (2011) Histopathologic diagnosis of fungal infections in the 21st century. Clinical microbiology reviews 24:247-280

9. Khot PD, Fredricks DN (2009) PCR-based diagnosis of human fungal infections. Expert review of anti-infective therapy 7:1201-1221

10. Kontoyiannis DP, Marr KA, Park BJ, Alexander BD, Anaissie EJ, Walsh TJ, Ito J, Andes DR, Baddley JW, Brown JM, Brumble LM, Freifeld AG, Hadley S, Herwaldt LA, Kauffman CA, Knapp K, Lyon GM, Morrison VA, Papanicolaou G, Patterson TF, Perl TM, Schuster MG, Walker R, Wannemuehler KA, Wingard JR, Chiller TM, Pappas PG (2010) Prospective surveillance for invasive fungal infections in hematopoietic stem cell transplant recipients, 2001-2006: overview of the Transplant-Associated Infection Surveillance Network (TRANSNET) Database. Clinical infectious diseases 50:1091-1100

11. Kraft C, Deplazes A, Sohrmann M, Peter M (2008) Mature ribosomes are selectively degraded upon starvation by an autophagy pathway requiring the Ubp3p/Bre5p ubiquitin protease. Nature cell biology 10:602-610 
12. Kume H, Yamazaki T, Togano T, Abe M, Tanuma H, Kawana S, Okudaira M (2011) Epidemiology of visceral mycoses in autopsy cases in Japan: comparison of the data from 1989, 1993, 1997, 2001, 2005 and 2007 in Annual of Pathological Autopsy Cases in Japan. Medical mycology journal 52:117-127

13. Montone KT, Guarner J (2013) In situ hybridization for rRNA sequences in anatomic pathology specimens, applications for fungal pathogen detection: a review. Advances in anatomic pathology 20:168-174

14. Neofytos D, Horn D, Anaissie E, Steinbach W, Olyaei A, Fishman J, Pfaller M, Chang C, Webster K, Marr K (2009) Epidemiology and outcome of invasive fungal infection in adult hematopoietic stem cell transplant recipients: analysis of Multicenter Prospective Antifungal Therapy (PATH) Alliance registry. Clinical infectious diseases 48:265-273

15. Nucci M, Perfect JR (2008) When primary antifungal therapy fails. Clinical infectious diseases an official publication of the Infectious Diseases Society of America 46:1426-1433

16. Rickerts V, Fredricks D (2012) Tissue Diagnosis of Invasive Fungal Infections: Current Limitations and the Emerging Use of Molecular Techniques. Curr Fungal Infect Rep 6:8

17. Rickerts V, Khot PD, Myerson D, Ko DL, Lambrecht E, Fredricks DN (2011) Comparison of quantitative real time PCR with Sequencing and ribosomal RNA-FISH for the identification of fungi in formalin fixed, paraffin-embedded tissue specimens. BMC infectious diseases 11:202

18. Rickerts V, McCormick Smith I, Mousset S, Kommedal O, Fredricks DN (2013) Deciphering the aetiology of a mixed fungal infection by broad-range PCR with sequencing and fluorescence in situ hybridisation. Mycoses im Druck

19. Rickerts V, Mousset S, Lambrecht E, Tintelnot K, Schwerdtfeger R, Presterl E, Jacobi V, JustNubling G, Bialek R (2007) Comparison of histopathological analysis, culture, and polymerase chain reaction assays to detect invasive mold infections from biopsy specimens. Clinical infectious diseases 44:1078-1083

20. Shoji JY, Kikuma T, Arioka M, Kitamoto K (2010) Macroautophagy-mediated degradation of whole nuclei in the filamentous fungus Aspergillus oryzae. PLoS ONE 5:e15650

21. Warner JR (1999) The economics of ribosome biosynthesis in yeast. Trends in biochemical sciences 24:437-440 